\title{
Intervenciones psicológicas en la prevención y atención a la dependencia
}

\section{Psychological interventions in prevention and attention to dependence}

Ignacio MONTORIO CERRATO*

\section{RESUMEN}

Lejos de la visión tradicional de la dependencia, el proceso que conduce a la dependencia a las personas de más edad tiene un carácter multicausal, de tal forma que trascendiendo a la dimensión biológica de la dependencia, los factores psicosociales adquieren un papel significativo. Existen múltiples vías de intervención procedentes de la Psicología de la Salud y la Psicología Clínica que debieran ser herramientas terapéuticas esenciales para la prevención y tratamiento de la dependencia. Asimismo, los cuidadores que proveen de asistencia a las personas mayores dependientes son uno de los factores psicosociales más influyentes a la hora de iniciar, mantener o reducir el proceso de dependencia y que deben ser atendidos, no sólo por ser agentes sociales que responden a la demanda planteada por el aumento del número de personas mayores dependientes que pueden contribuir al mantenimiento de su autonomía, sino por las consecuencias negativas que para ellos mismos puede tener el mantenimiento de tal actividad.

\section{PALABRAS CLAVE}

Depèndencia, Tratamiento psicológico, Personas mayores.

\section{ABSTRACT}

Far from the traditional view of care need, the process leading to the need of care in elder persons is multi-causal. Beyond the biological dimension, psycho-social factors play

\footnotetext{
*Facultad de Psicología. Universidad Autónoma de Madrid. ignacio.montorio@uam.es
} 
a significant role. Health Psychology and Clinical Psychology provide ways of intervention along with prevention and treatment tools. Persons providing care to hte elderly play a key role within the social process of care work. They are not only social agents,trying to meet increasing social needs of elder persons, helping them to gain autonomy but they also have to avoid the negative implications of their practice.

\section{KEY WORDS}

Care need, Psychological intervention, Elder persons. 


\section{LA IMPORTANCIA DE LA INDEPENDENCIA}

La adaptación de las personas mayores a su medio y su bienestar está, primordialmente, ligado a la capacidad que tengan para llevar a cabo las actividades cotidianas esenciales. El número y calidad de años que una persona mayor puede vivir sin discapacidades llevó al desarrollo de la noción de "expectativa de vida autónoma”, considerado por la OMS como un índice fundamental de la salud y, por extensión, de la calidad de vida de una población (OMS, 1984). La autonomía personal consiste básicamente en el control sobre la toma y ejecución cotidiana de decisiones cuya pérdida afecta gravemente tanto al individuo como al entorno, ya que la capacidad de funcionar independientemente es lo que se considera estadísticamente más frecuente y normativamente más deseable (en los trabajos de análisis de discurso, el mantenimiento del "estilo de vida propio" es la meta más valorada por las personas mayores (Bazo, 1992). Además el miedo más frecuente de las personas mayores es llegar a tener que depender de los demás (INSERSO, 1995). Así mismo, se ha demostrado que existe una estrecha relación entre la realización de actividades cotidianas y la autoestima de las personas mayores (Reitzes, Mutran y Verrill, 1995), así como entre la capacidad para realizarlas de forma independiente y la percepción de autoeficacia (Willis, Jay, Diehl y Marsiske, 1992). Por otra parte, el fenómeno de la dependencia supone que las necesidades básicas que el individuo no puede satisfacer por sí mismo deben ser cubiertas por el entorno 0 , más concretamente, por aquellas personas próximas al individuo de las que se espera y/o exige que realicen esa labor de asistencia y cuidado. En este sentido, se puede considerar que las consecuencias más importantes de la pérdida de autonomía personal son tanto de tipo personal, al verse afectados negativamente el autoconcepto y el bienestar autopercibido, como de tipo socioeconómico, ya que la necesidad de la prestación de cuidados debe cubrirse por la familia, con los subsiguientes cambios en las rutinas, necesidades y relaciones de sus miembros, o por profesionales, con el subsiguiente desembolso económico por parte del individuo y su familia 0 de ayuntamientos, comunidades o el estado.

\section{PÉRDIDA DE AUTONOMÍA Y DEPENDENCIA}

La principal consecuencia de una pérdida en la autonomía personal es la necesidad del individuo de ser ayudado 0 apoyado para ajustarse a su medio e interaccionar con él. Así, desde una aproximación funcional, se puede definir la dependencia a partir del ajuste de los individuos a las demandas de su medio fisico y social. Este ajuste hace referencia al funcionamiento en una serie de áreas que integran la vida cotidiana en la sociedad occidental actual, siendo las principales la dependencia económica, la dependencia fisica, que tiene lugar cuando el individuo pierde el control de sus funciones corporales y de su interacción con los elementos físicos del ambiente, la dependencia social, que se asocia con la pérdida de las personas y relaciones significativas para el individuo y la dependencia mental, que aparece a medida que el individuo pierde su capacidad para resolver sus problemas y tomar decisiones propias.

Generalmente, la dependencia se ha limitado al área física, para evaluar el nivel de dependencia y el tipo y grado de cuidado necesario para paliar dicha dependencia. Así, en los estudios epidemiológicos el término dependencia suele definirse como "incapacidad funcional" 
en relación al conjunto de conductas que una persona ejecuta todos los dias o con frecuencia casi cotidiana, para vivir de forma autónoma e integrada en su medio ambiente y cumplir con su rol social y que ha venido a denominarse actividades de la vida diaria. De esta forma, una persona es dependiente cuando presenta limitaciones en la ejecución de una 0 más actividades, consideradas componentes básicos de la vida diaria, y que, por lo tanto, necesita depender de otra persona.

\section{LA VISIÓN ACTUAL DE LA DEPENDENCIA}

El significado de la dependencia en referencia a la edad avanzada parece a la luz de lo mencionado anteriormente sencillo. Se piensa habitualmente en la dependencia como un fenómeno que refleja la pérdida de autonomía personal cuya principal consecuencia es la necesidad por parte de la persona mayor de ser ayudada 0 apoyada para ajustarse a su medio e interactuar con él. Además la pérdida de autonomía personal es definida, casi con exclusividad, en términos físicos o biológicos ligado principalmente al padecimiento de problemas de salud, lesiones como roturas de cadera y las enfermedades neurodegenerativas -demencias- que impiden actuar de una forma similar a antes de la aparición de la enfermedad. Asimismo, la dependencia es explicada como una consecuencia natural del paso de los años.

En definitiva, la dependencia suele ser entendida como un fenómeno unidimensional, es decir, un declive físico esperable de mayor o menor cuantía. Una muestra de ello es la definición pragmática de dependencia que habitualmente utilizan los estudios epidemiológicos que intentan cuantificar el alcance de la dependencia, haciendo sinónimos "dependencia" e "incapacidad funcional en relación a las actividades de la vida diaria”. Esta habitual visión de la dependencia es fácilmente asumible ya que a simple vista se observa que la dependencia física entre las personas mayores aumenta con la edad demandándose 0 aceptándose una mayor cantidad de ayuda proveniente de los demás. Menos intuitivo y socialmente aceptado es el reconocimiento de cómo determinados aspectos psicológicos o el entorno social de las personas afecta a la dependencia

\section{LA DEPENDENCIA COMO UN FENÓMENO MULTICAUSAL}

Desde una amplia perspectiva, esta concepción unidimensional de la dependencia no debería ser la única, sino que es preferible pensar en la dependencia como un concepto de naturaleza compleja, con diversas caras, en el que influyen variables biológicas, psicológicas y sociales, siendo la intervención de todos estos aspectos el objeto principal de este trabajo. La dependencia presenta múltiples facetas y dimensiones. Esto se refleja en la amplia gama de conceptos de dependencia que aparece en la literatura: dependencia mental, física, económica, social, emocional, cognitiva, imaginaria, neurótica, funcional, etc. Asimismo, la dependencia puede ser analizada según distintas perspectivas: conductual, personal, situacional e interpersonal. En resumen, puede concluirse que los distintos enfoques generalmente se centran en aspectos específicos de la dependencia y raramente la consideran en toda su complejidad (Horgas, Wahl y Baltes, 1996).

También, el estudio de la dependencia admite distintos niveles de análisis de mayor o menor grado de generalidad. Así, es posible plantearla en términos globales o de macronivel, utilizándose el 
concepto de dependencia para reflejar la estructura demográfica de un país (por ejemplo, la ratio de dependencia); un nivel de análisis intermedio de dependencia reflejaría, por ejemplo, datos referidos a ratios de necesitad ayuda para cubrir necesidades especificas de AVD en contextos delimitados (p.ej., centros residenciales. Estos dos niveles de análisis, global e intermedio, son los más habitualmente utilizados para el análisis de la dependencia. Pero, también es posible, finalmente, un micronivel de análisis de la dependencia, persona a persona, que sería aquel que permite caracterizar la conducta dependiente del individuo interactuando con su ambiente social inmediato. Este nivel de análisis determinaria, por tanto, que la dependencia es función de tres factores; las características de cada persona mayor, el contexto social en el que se produce la conducta dependiente y la interacción entre ambos 0 , lo que es lo mismo, este nivel de análisis permite describir la cantidad y naturaleza de dependencia que es socialmente inducida. Este nivel de análisis ha sido el menos analizado y sobre el que aún no se han propuesto suficientes programas o protocolos de intervención. Unicamente, el micronivel permite conocer la influencia de las variables no biológicas sobre la dependencia $\mathrm{y}$, por tanto, planificar intervenciones correctoras de prevención o paliamiento de la dependencia.

En última instancia, la idea que se presenta acerca de la dependencia es la de reflejar ésta como un constructo multicausal donde los factores biológicos, psicológicos y sociales son todos ellos antecedentes que inducen a la dependencia conductual, esto es, a solicitar o aceptar pasiva o activamente la ayuda de los demás (Baltes y Wahl, 1990). Veamos los principales factores que causan dependencia que, a su vez, son las vias de intervención ante la misma.

\section{Factores físicos}

Fragilidad fisica. El deterioro de alguno o varios de los sistemas biológicos del organismo (cardiovascular, respiratorio, muscular, etc.) provocan un deterioro de la fuerza física, movilidad, equilibrio, resistencia, etc. que se asocia a una disminución de la ejecución de actividades básicas e instrumentales de la vida diaria. Aun siendo cierto este declive, es necesario recordar las amplias diferencias individuales entre las personas mayores, así como que la capacidad para modificar el declive biológico demorar, prevenir e, incluso, re-establecer- ha sido ampliamente infravalorado (Rowe y Kahn, 1987). Existe hoy un importante cuerpo de investigación que evidencia los beneficios de intervenciones que pueden mejorar el funcionamiento físico y así reducir la dependencia. La práctica del ejercicio físico en distintas intensidades es un importante predictor de morbilidad y longevidad. En relación a ello, uno de los principales retos es implicar a las personas adultas en la actividad física, siendo las variables motivacionales y disposicionales un ingrediente importante.

Enfermedades crónicas. Indudablemente, el padecimiento de las enfermedades físicas crónicas durante la vejez (por ejemplo, osteoporosis, osteoartritis, ACV, etc.,) y las limitaciones sensoriales contribuyen de forma notable a la discapacidad y dependencia física. Como sucede con la fragilidad física el papel de la prevención en la aparición de las enfermedades crónicas que pueden retrasar o disminuir la severidad de la dependencia es infravalorado. Las intervenciones habitualmente empleadas en el marco de la Psicología de la Salud para la prevención y tratamiento en la diabetes, accidentes cerebrovasculares, infartos, etc. serían un elemento de tratamiento principal. 
Utilización de fármacos. La alta prevalencia de enfermedades entre las personas mayores provoca un elevadísimo consumo de fármacos que conllevan en ocasiones unos importantes efectos secundarios, así como interacciones farmacológicas no deseadas (por ej., Vestal, 1990). Por ejemplo, la confusión, el deterioro cognitivo, el aplanamiento afectivo, etc. son sintomas conductuales derivados del consumo de fármacos que se encuentran con relativa frecuencia entre las personas mayores, factores que tienden a aumentar la dependencia física y contribuyen a la dependencia conductual (Horgas et al., 1995). No sólo un mayor control sanitario de las prescripciones farmacológicas y de sus efectos secundarios puede redundar en beneficio de una menor dependencia, sino que las intervenciones para una adecuada adherencia terapeútica son claves.

\section{Factores psicológicos}

Trastornos mentales. Algunos trastornos tales como la ansiedad y, especialmente, la depresión contribuyen significativamente a la dependencia en la edad avanzada. Es frecuente entre las personas mayores una elevada sintomatología de depresión que, a menudo, no es diagnosticada ni tratada. No obstante, es bien conocido que la sintomatología depresiva se asocia a aislamiento social, quejas fisicas, declive cognitivo y funcional, factores todos ellos que contribuyen a la dependencia conductual (Frazer, Leicht y Baker, 1996). Especialmente importante es el caso de las personas con enfermedad de Alzheimer ya que se calcula que, aproximadamente, al menos un tercio de las personas que la padecen en sus estadios iniciales y medios pueden sufrir problemas de depresión (Teri y Gallagher, 1991).
Dolor. El padecimiento de dolor es una de las dimensiones psicológicas que más contribuyen a la dependencia, no sólo por la disminución directa de la actividad, sino por el efecto indirecto que provoca sobre el aparato osteomuscular como consecuencia de la disminución de la actividad que, a su vez, genera mayor dolor. Asimismo, existe un círculo vicioso entre el dolor, depresión y pérdida funcional. Se ha demostrado que las técnicas de intervención habituales para el tratamiento del dolor son útiles también en población mayor.

Caídas y miedo a las Caídas. De manera similar a lo anterior, el haber padecido una caída o, simplemente, el miedo a caerse supone una importante fuente de dependencia como consecuencia de la reducción de la actividad. El entrenamiento supervisado en educación, balance y equilibrio postural, ejercicio de fortalecimiento muscular, control de medicamentos, modificación de "peligros" ambientales, la psicoeducación y las técnicas habituales del tratamiento de la ansiedad son efectivas para el tratamiento de este problema.

Factores de personalidad. Las experiencias y aprendizajes a lo largo de la vida hacen que al alcanzar la vejez las personas difieran notablemente en cuanto a sus demandas y aceptación de ayuda de los demás ante las distintas situaciones de la vida cotidiana, mostrándose más 0 menos dependientes. Además, esta característica de personalidad dependiente puede aumentar el riesgo de padecer trastornos de salud fisica y mental y, por tanto, indirectamente aumentar la dependencia (Greenberg y Bornstein, 1988).

\section{Factores contextuales}

Ambiente fisico. La investigación, la 
práctica profesional y la experiencia común de muchos familiares de personas mayores muestra, sin lugar a dudas, que un ambiente físico estimularmente rico con suficientes ayudas protésicas, que conjugen adecuadamente la autonomía con la seguridad, contribuyen a que las personas mayores funcionen en unos niveles de ejecución óptimos. Por el contrario, un ambiente poco estimulante 0 sin suficientes ayudas contribuye al incremento de la dependencia conductual .

Ambiente social. Un último tipo de antecedentes o causas que generan dependencia se encuentran en el contexto en el que se produce la dependencia, en el ambiente social que favorece 0 , por el contrario, previene la dependencia Entre los diversos factores de este tipo, probablemente, dos de ellas sean las más importantes:

\section{a) Las contingencias ambientales.}

Se basa en el modelo de aprendizaje operante, desde el cual las conductas dependientes de las personas mayores son entendidas y explicadas como conductas instrumentales. Este paradigma ha generado una amplia cantidad de trabajos de investigación cuya máximo exponente lo podemos encontrar en los trabajos del equipo de Margaret Baltes (p. ej., Baltes, 1988). Se ha observado una serie de patrones de interacción que se establecen con cierta frecuencia entre la persona mayor y sus cuidadores durante diversas actividades en multitud de contextos cotidianos, desde instituciones de cuidados especiales hasta la propia vivienda. Obviamente, modificando las contingencias ambientales que siguen a la manifestación de conductas dependientes se ha logrado disminuir las conductas dependientes, esto es, enseñando a las personas que interaccionan con los mayores a responder positivamente ante conductas de autonomía e independencia y, por el contrario, no reforzar las conductas dependientes se ha conseguido reestablecer, por ejemplo, hábitos relacionados con el autocuidado (higiene, comida 0 vestido) o disminuido conductas de dependencia relacionadas con la deambulación, las alucinaciones, la depresión o la incontinencia.

\section{b) Expectativas y estereotipos}

Por último, las expectativas sociales acerca de las personas mayores son muy probablemente un factor principal en la producción de la dependencia. Los gerontólogos han acuñado el término "edadismo" para referirse al concepto peyorativo de juzgar, clasificar o describir a alguien basado en su avanzada edad cronológica. La vejez es consecuencia de un proceso biológico, pero es también una construcción cultural (Beauvoir, 1970). Una persona es vieja, como en cualquier otro rol y estatus social, cuando las demás personas así la consideran. En el proceso de la interacción social, las demás personas, a través de sus conductas, son el espejo en el que uno mismo se ve reflejado. Las reacciones de los demás hacía una persona le muestran la imagen que presenta, constriñéndola a adoptar los comportamientos que sabe que esperan de ella. Se ha sugerido que el edadismo se mantiene por las falsas creencias que socialmente imperan sobre la vejez, influyendo no sólo en el modo cómo se trata a las personas mayores, sino también en el modo en que ellas se comportan. La figura $\mathrm{x}$ muestra un modelo con el siguiente proceso de interacción entre, por un lado, los estereotipos y expectativas negativas sobre la vejez y, por el otro, la dependencia para las actividades de la vida diaria. 
Intervenciones psicológicas en la prevención y atención a la dependencia

Figura 1. Mecanismos de influencia sobre la dependencia
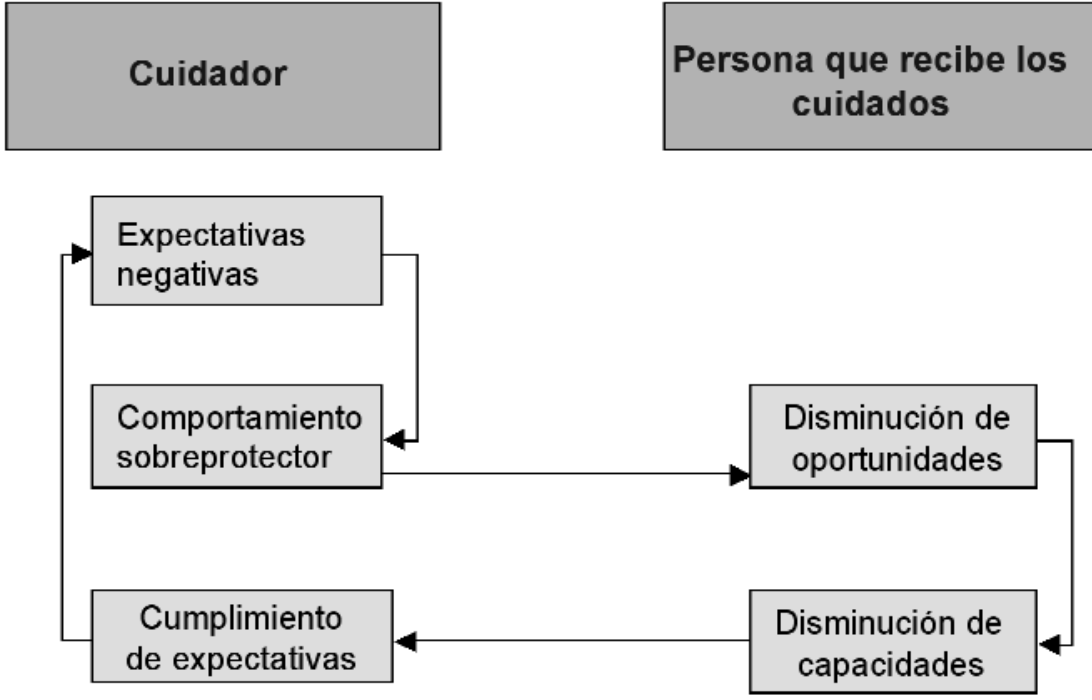

\section{EXCESO DE INCAPACIDAD Y DEPENDENCIA}

Tal como defienden Baltes y Baltes (1990), al ser la dependencia funcional un fenómeno multicausal, si bien es indudable que existen una serie de condicionantes orgánicos estables, debemos resaltar la importancia de estudiar el peso de factores potencialmente modificables, así como la posibilidad de intervenir sobre ellos para evitar lo que se denomina en la literatura gerontológica exceso de incapacidad.

Así, el exceso de incapacidad se refiere al deterioro provocado por el desuso de capacidades preservadas que no son consecuencia directa de un estado de fragilidad física 0 enfermedad y que son de carácter reversible. El exceso de incapacidad significa atribuir a la enfermedad los comportamientos de dependencia cuando de hecho se derivan también de otra fuente más benigna que si es reversible (efectos secundarios, alteracio- nes de conducta, ambiente fisico inadecuado, contingencias ambientales favorecedoras de dependencia o estereotipos y atribuciones negativas hacia las personas mayores) (ver figura 3).

\section{La atención a los cuidadores como vía para el fomento de la independencia}

Los cuidadores informales que proporcionan aproximadamente el $86 \%$ del cuidado que se presta en el domicilio (INSERSO, 2004) son la principal influencia psicosocial en la atención a los mayores. Atender a los cuidadores y hacer frente a los obstáculos que éstos pueden encontrar a la hora de atender a sus familiares no son sólo un objetivo legitimo de intervención ante el fenómeno de la dependencia entre las personas mayores, sino un objetivo expresado en la propia Ley Básica sobre Autonomía Personal y Protección a las Personas en Situación de Dependencia que incluye desarrollar un sistema de atención inte- 
Ignacio Montorio Cerrato

Figura 2. Exceso de discapacidad

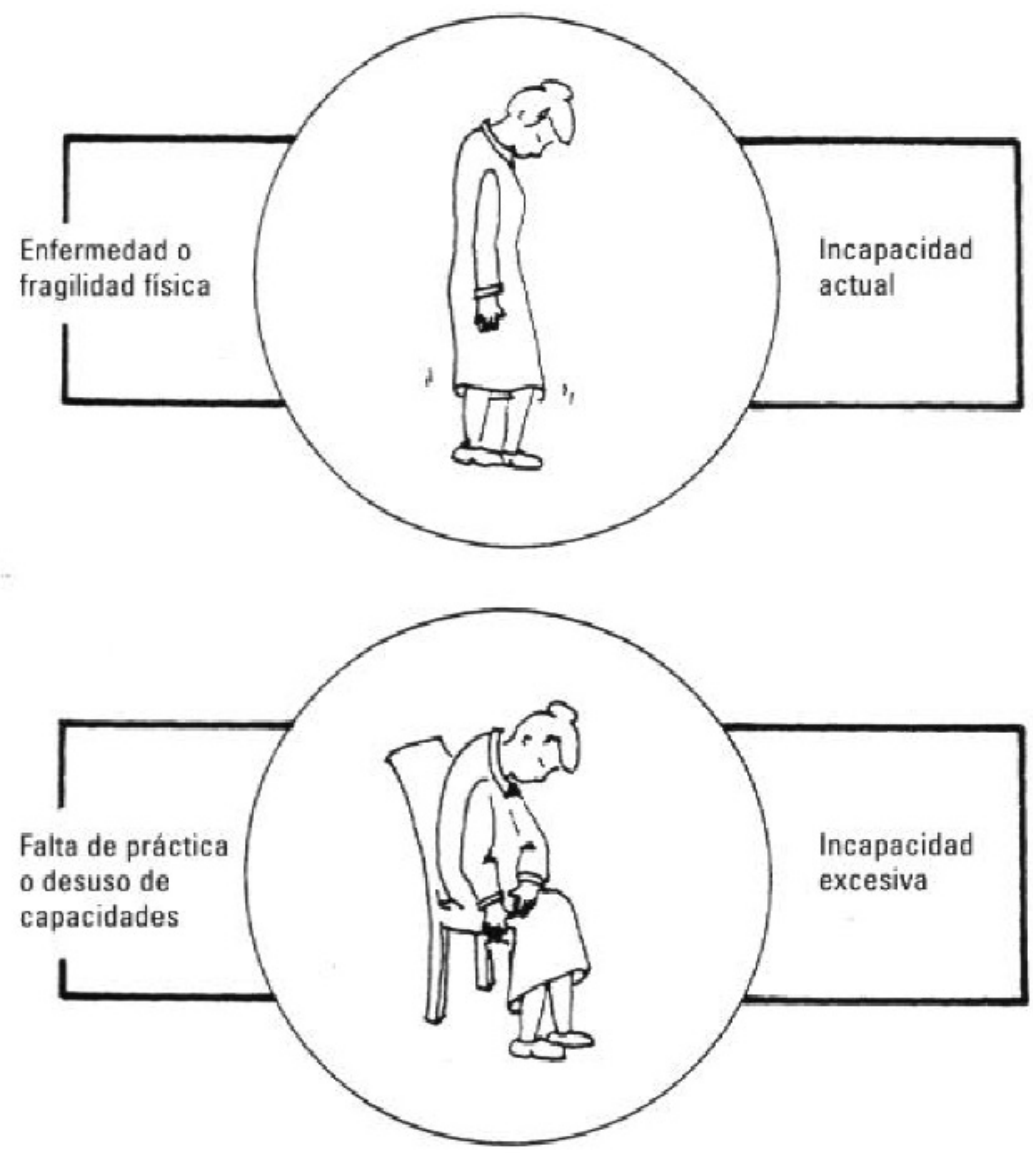

gral e integrada para las personas mayores y sus familias, asi como elaborar y aplicar programas de cuidado a cuidadores. Así, la figura del cuidador no debe ser considerada meramente como un recurso para responder a las necesidades de las personas mayores, sino que debe considerarse como un objetivo principal de la atención sociosanitaria (García-Calvente, 2000).

La atención a los cuidadores reúne dos vías de intervención diferenciadas: la formación a los cuidadores como agentes sociales que favorecen la autonomía y previenen la dependencia de sus familiares y las intervenciones dirigidas a paliar las consecuencias negativas del cuidado.

En primer lugar, las intervenciones psicoeducativas con cuidadores reduce la dependencia para actividades instrumentales. Se considera que un desequilibrio entre la competencia de los individuos y las demandas del ambiente produce problemas en el funcionamiento y en el bienestar. Los programas desarrollados para ayudar a cuidadores familia- 
Intervenciones psicológicas en la prevención y atención a la dependencia

\section{Figura 3. Recomendaciones generales de actuación ante una situación de dependencia}

- Acondicionar el entorno de la persona mayor para facilitar conductas independientes (poner un plato de ducha, poner carteles o signos identificativos en caso de dificultades cognitivas -ej., baño-, iluminación adecuada, etc.).

- Observar a la persona mayor para conocer todo lo que puede hacer por sí solo y no hacer nada por la persona que ésta pueda hacer por sí misma. Ayudar solo en lo necesario.

- Preparar la situación para que sea más fácil ser autónomo.

- Comportarse con la persona mayor de modo que favorezca la autonomía: reforzar las conductas autónomas.

- Responder adecuadamente cuando la persona pone objeciones a los intentos para favorecer la autonomía (animar, demostrar confianza en su capacidad, ser persistente).

- Potenciar la autoestima de la persona dependiente (que pueda tomar decisiones, por pequeñas que éstas sean, empezar por tareas o actividades sencillas que se sepan hacer). No se debe olvidar que, para que estas técnicas funcionen, el cuidador (formal e informal) ha de tener las siguientes características: paciencia, flexibilidad, sensibilidad, amabilidad, creatividad y, por encima de todo, interés real por potenciar la autonomía de las personas mayores.

res a reducir este desequilibrio les proporciona habilidades y herramientas que les permiten manipular de manera efectiva las dimensiones del entorno domiciliario asociadas con los problemas diarios asociados al cuidado. Algunas de estas estrategias que se trabajan con los cuidadores están dirigidas a modificar la dimensión física del ambiente (por ej., barras asideras, ambientes sencillos mediante eliminación de objetos, etc.), otras están dirigidas a alterar la dimensión de la tarea (por ej., formas de comunicación eficaces) y otras están dirigidas a modificar el ambiente social (coordinación de las actividades del cuidado, instrucciones sobre cómo comunicarse con los cuidadores formales, etc.). En un programa especifico con estos componentes de intervención se encontró que tras la intervención la dependencia para actividades instrumentales declinaba significativamente menos en el grupo experimental que en el control y, para las actividades básicas, se encuentra una tendencia en el mismo sentido aunque no llega a ser significativa (Gitlin y otros, 2001). Asimismo, se ha contrastado que intervenciones conductuales complementadas con ejercicio mejora de manera significativa la salud física y la depresión de personas con Alzheimer. Un programa de intervención dirigido a entrenar a cuidadores a identificar y modificar los problemas conductuales de las personas con Alzheimer y a promover la realización de un mínimo diario de 30 minutos de ejercicio (por ej., aerobic y flexibilidad) mejoraba de manera significativa la salud fisica y la depresión de personas con Alzheimer. Las personas que participan en la intervención man- 
tienen durante dos años un mayor rendimiento en funcionamiento físico y menor depresión que otras personas con Alzheimer que reciben los cuidados habituales (Teri y otros, 2003). En la figura 3 se incluyen algunas rrecomendaciones generales de actuación ante una situación de dependencia.

En segundo lugar, la combinación de servicios de respiro junto a la implantación de intervenciones psicoeducativas son una de las opciones más eficaces para ayudar a los cuidadores (Díaz, Montorio y Yanguas, 1999). Estas implican un programa de actuación estructurado, orientado a objetivos específicos, en grupo, dirigidos por uno o más profesionales, a través de los cuales se pretende proporcionar a los cuidadores información sobre la enfermedad de sus familiares y sobre recursos y servicios disponibles en la comunidad y sobre como cuidar mejor de sus familiares y de ellos mismos.

\section{CONCLUSIONES}

Una parte de la dependencia funcional puede ser explicada a partir de una incapacidad funcional objetiva, como un deterioro cognitivo severo o una pérdida de movilidad por el padecimiento de determinados procesos mórbidos, aunque, incluso en estos casos la dependencia puede ser prevenida y paliable en su grado de intensidad con tratamientos adecuados. Otra parte de la dependencia puede ser explicada a partir de variables y procesos psicosociales como las alteraciones de conducta, como la depresión o algunos sintomas de enfermedades como los asociados a la demencia que son tra- tables (alucinaciones, deambulación, etc.), el ambiente físico y los mecanismos adaptativos de la persona mayor ante determinadas contingencias ambientales, la continua interacción existente entre variables psicosociales tales como los estereotipos, las atribuciones, las actitudes, las conductas de apoyo y de comunicación, la autoeficacia que pueden llegar a inducir dependencia y son compartidas por la sociedad. En definitiva, la dependencia es un fenómeno, como otros muchos, complejo, que admite muchas vías de intervención como las habituales referidas a las prestaciones sociales más clásicas -servicios, ayudas económicas-. Pero también se hacen imprescindibles otras intervenciones cuando las conductas de dependencia son el resultado de una conformidad con los estereotipos, una subestimación de los recursos propios, de las contingencias ambientales o de una ambiente físico no facilitador. Finalmente, los procesos que conducen a la dependencia son multivariados en sus trayectorias de tal forma que un buen número de intervenciones psicológicas pueden convertirse en pequeños ingredientes que retrasan, palian o evitan los procesos que conducen a la dependencia. Cuando una persona mayor padece un problema osteomuscular no debe ser un sinónimo de dependencia, sino que un adecuado control médico, junto con intervenciones que procuren la realización de actividad física, programas de prevención de aparición de la depresión, programas multidisciplinares del tratamiento del dolor y de prevención de caídas y un entorno social que participe y colabore activamente en el mantenimiento de la autonomía son vías reales y contrastadas para la prevención de la dependencia. 
Intervenciones psicológicas en la prevención y atención a la dependencia

\section{BIBLIOGRAFÍA}

Baltes, M.M. (1988). The etiology and maintenance of dependency in the elderly: Three phases of operant research. Behavior Therapy, 19, 301-319.

Bazo, M.T. (1992). La ancianidad del futuro. Barcelona: SG. Editores.

Díaz-Veiga, P., Montorio, I. y Yanguas, J. (1999). Intervenciones con cuidadores de personas mayores. En M. Izal e I. Montorio (eds), Gerontología Conductual. Bases para la intervención y ámbitos de aplicación. Madrid: Síntesis.

Frazer, d.w., Leicht, M.L. y Baker, M.D. (1996) En L.L. Carstensen, B.A. Edelstein y L. Dornbrand (Eds.), The practical handbook of clinical gerontology. Thousand Oaks: Sage Publications, 1996.

García Calvente (2000). El Sistema Informal de Atención a la Salud. Conferencia impartida en el XXIX Congreso Nacional Ordinario de Pediatría. Tenerife.

Gitlin, L.N., Corcoran, M., Winter, L., Boyce, A and Hauck, W.W. (2001). A Randomized, Controlled Trial of a Home Environmental Intervention: Effect on Efficacy and Upset in Caregivers and on Daily Function of Persons With Dementia. The Gerontologist, 41(1), 4-14.
Horgas A.L., Wahl, H.W. y Baltes, M.M. (1996). Dependency in Late Life. En L.L. Carstensen, B.A. Edelstein y L. Dornbrand (Eds.), The practical handbook of clinical gerontology. Thousand Oaks: Sage Publications, 1996.

MSERSO (2004). Las personas mayores en España. Informe 2004 Madrid: Imserso.

Reitzes, D.C., Mutran, E.J., y Verrill, L.A. (1995). Activities and self-esteem. Research on aging, Vol. 17 (3), 260-277.

Rowe, J.W. y Kahn, .L. (1987). Human aging: Usual and succesful. Science, 233, 1271-1276.

Teri, L., Gibbons, L.E., McCurry, S.M., Logsdon, R.G., Buchner, D.M., Barlow, W.E., Kukull, W.A., LaCroix, A.Z., McCormick, W. Y Larson, E.B. (2003). Exercise Plus Behavioral Management in Patients With Alzheimer Disease. A Randomized Controlled Trial. Journal of the American Medical Association, 290(15), 2015-2022.

Willis, S.L., Jay, G.M., Diehl, M. y Marsiske, M. (1992). Longitudinal change and prediction of everyday task competence in the elderly. Research on aging, Vol. 14 (1), 68-91. 